\title{
The Finite Element Analysis of the Clip Type Prestressed Anchor
}

\author{
Ding Kewei ${ }^{1,}$, Liu Yawen ${ }^{2, b}$ \\ ${ }^{1}$ School of Civil Engineering Anhui Jianzhu University, Hefei 230601, China \\ ${ }^{2}$ School of Civil Engineering Anhui Jianzhu University, Hefei 230601, China \\ aemail: dingkewei@ahjz.edu.cn, bemail:hh0312lyw@126.com
}

Keywords: Prestressed; Clips; Anchorage; Fnite Element Analysis

\begin{abstract}
With the development of society and economy, to meet the people's life,the urban construction is to rise.Amongthem, as an important part of civil structures, prestressed structure with good technical and economic indexes and the widespread usage is widely used in various fields of urban construction.Being an important component of prestressed anchor and prestressed anchorage system, whose bearing capacity has a direct impact on the effect and safety of prestressed structure.In this paper, in the light of the application of prestressed anchorage system in the project, with the single hole anchorage as the research object, we study on the anchorage performance of the anchorage through the analysis of the Ansys finite element method.To set the contrast model group by changing the parameters, we get the stress and strain distribution of different anchorage parameters group. The results obtained are analyzed model, insert the relationship between film thickness and stress tolerance and gives a reasonable analysis.
\end{abstract}

\section{Introduction}

Clip type single hole prestressed anchorage is the key part of the construction technology of prestressed concrete, which is widely used in large span railway, highway bridges, industrial buildings, dams, nuclear reactor shell and other special buildings.As a comprehensive technology across the two disciplines of mechanical and civil engineering, the prestressing force can not only increase the bearing capacity of the structure, but also save material and prolong the service life of the structure.In addition, the anchorage also gets more and more attention as the key part to ensure the efficient transfer of prestressing force.

Clip type single hole anchorag is composed of the anchor ring, clip, the anchor plate and some other pressed elements.Among them, the clip and the anchor ring are the important anchoring units[1].Clip Type Anchors generally use high Strand retracted into the clip to achieve the anchor.There is no need of a top plug when the anchor works, which belongs to a self anchored anchorage[2]. The anchoring performance of anchorage is stable, the stress distribution is more uniform and reliable.Besides, it has a wide range of steel stand anchorage.

Due to the complexity of anchorage force, if we use the traditional method, the process will be complicated and the result will be not precise enough, so it can be carried on the simulation analysis by using the finite software.ANSYS finite element analysis software provides the feasibility analysis of anchorage of prestressed work.With the help of the software,we make use of the symmetry of the structure to analysis the target unit.We analysis and calculate the model by pressurizing on the entity, mainly for the stress and strain of anchorage changing with the thickness of the anchor clip,which also means the change of inner and outer diameter of the end of clip. With this method, we provide reasonable theoretical support for the optimization of anchorage[4]. 


\section{Dimensions and parameters of the single hole anchorage}

There is a two hole anchor type clip anchorage steel strand to form a unit in an anchorage[3].The mechanical properties of each unit in porous anchorage are the same. We can convert the pressure from the sleeve to the friction between the working cone and the sample by the deformed cone which is squeezd by the mechanical pressure from the sleeve[4]. When the sample is pulled, the friction value of the sample and the working cone can be detected, and the maximum tensile force of the device is obtained[5].The phenomenon is called the more wedge there is, the tighter there will be.Anchoring principle diagram is as follows:

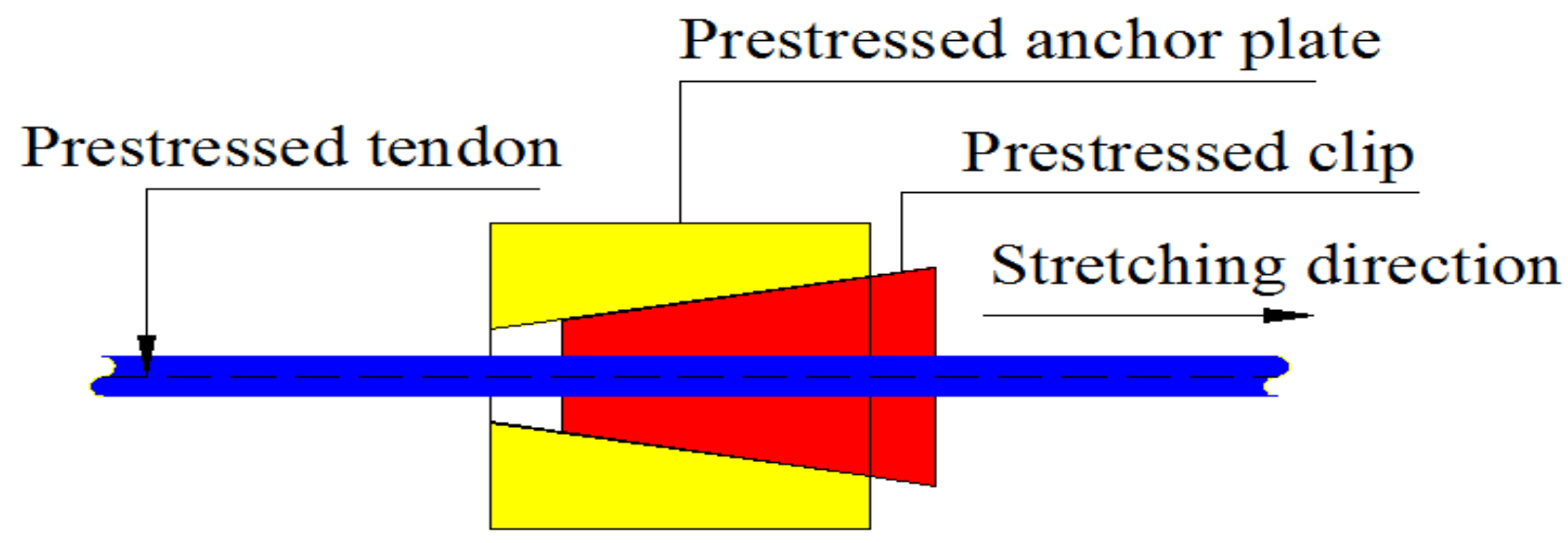

Fig.1.The anchoring principle diagram

To set up the finite element analysis model of the single hole anchorage of prestressed shown in Fig.1, we take the clip thickness as the parameter variable under the condition of the constant taper, board length, board thickness and the terminal inner diameter of the clip to change outer diameter of the end so that the thickness of the clip changes.Anchorage Model material parameters of the components is as shown in Table 1.Parameter settings sees Table2.

Table.1.The material parameters of model

\begin{tabular}{|c|c|c|c|}
\hline Member name & $\begin{array}{c}\text { Modulus of elasticity } \\
(\mathrm{E}) \mathrm{MPa}\end{array}$ & $\begin{array}{c}\text { Poisson's } \\
\text { ratio }(\mu)\end{array}$ & $\begin{array}{c}\text { Friction } \\
\text { coefficient (f) }\end{array}$ \\
\hline Sleeve & $1.95 \mathrm{e} 5$ & 0.3 & 0.2 \\
\hline Working Cone & $2.05 \mathrm{e} 5$ & 0.3 & 0.2 \\
\hline Sample & $1.9 \mathrm{e} 5$ & 0.3 & 0.2 \\
\hline
\end{tabular}

Table.2.The parameter setting

\begin{tabular}{|c|c|c|c|}
\hline Parameter & Meaning & Parameter & Meaning \\
\hline theta $=3$ & Taper & $\mathrm{uu}=0.3$ & Right sleeve interference fit \\
\hline long $=70$ & Length of the working cone & $\mathrm{xx}=30$ & Claping length of the sample \\
\hline $\operatorname{din}=4.75$ & Inner radius of the working cone end & $1 \mathrm{~s}=80$ & $\begin{array}{l}\text { Distance to the origin from the } \\
\text { right of the sample }\end{array}$ \\
\hline dout $=11$ & Outer radius of the working cone end & $\mathrm{nn}=9$ & Gap angle of the cone \\
\hline $11=60$ & Depth of the working conee & $\mathrm{fknn}=1$ & Stiffness factor \\
\hline $1 \mathrm{~m}=25$ & Length of the sleeve & step $1=20$ & Steps of the first load step \\
\hline $\mathrm{dd}=5$ & Initial length of the sleeve end & step2 $=50$ & Steps of the second load step \\
\hline
\end{tabular}




\section{The assumptions and models}

In ANSYS, the interference problem is about contaction, which is mainly a highly non-linear behavior.For the tapered collet of the prepressed anchorage is composed of virous components and there is similar rigidity in each other, the flexibility - type soft contact can be used.The contact among the sleeve, working cone and the sample in the compent belongs to face - face[2].In ANSYS, the target and contact elements form a "contact pair."In the simulation, in order to facilitate the network division, we choose unit MESH200 in the plane model whose element shape is a quadrangle, while sleeve, the working cone and sample will be dealt with unit SOLID185 in entity, unit CONTA173 in contact and unit TARGE 170 in the target unit[5][6].

The loading steps are as follows[7]:

(a)Do the analysis of the interference fit between the sleeve and the working cone.

(b) Take the sample out and do the analysis of contact friction.

Set up the computational hypothesis of the finite element mode[2]:

(a)For conical clamping in the work of the component, material nonlinearity is not considered and the components are in elastic state .

(b)Since the assembly process is not concerned with the sleeve and the working cone, the interference fit is directly considered in the model.

(c)For the analysis of friction conical clamping provided, the change of joint forces of the end nodes along the axis of the specimen is considered the change of friction between the specimen and the working cone when it is in the process of taking out the sample.

(d)The first load step constraints on the left side of the work cone section node, the right end of the specimen and the outer surface of the sleeve node, the second load step to release the right side constraints and the remaining unchanged .

Equally divide the finite element mesh of the anchorage though ANSYS finite element method under the circumstances of different clip's thicknesses so that the stress cloud can be obtained[1].Different colors show different stress regions. With the observation of stress, strain and displacement in different clap's thickness groups by stress cloud, we analysis the data and draw conclusions[3].Fig 2 shows the caltulation unit.The anchorage taper is 3 degree, cone length is $70 \mathrm{~mm}$, the sample at the end of the inner radius is $4.75 \mathrm{~mm}, \mathrm{~h}$ is the height of contact piece between the clap and the sample and outer radius ranges from $8.75 \mathrm{~mm}$ to $14 \mathrm{~mm}$ with the change interval by $0.375 \mathrm{~mm}$.

ELagerts

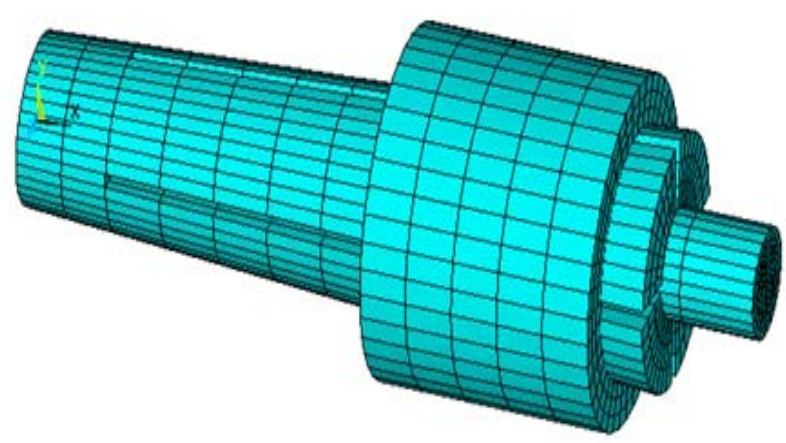

Fig.2.Finite element modeling of anchorage
NN

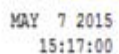

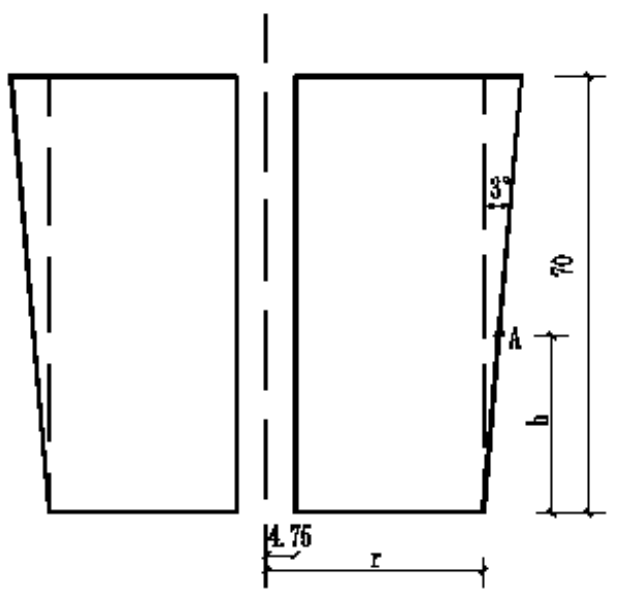

Fig.3.The single hole anchorage calculation unit schematic 
Calculation and analysis of the structure

\begin{tabular}{|c|c|c|c|c|c|c|}
\hline \multirow{3}{*}{$\begin{array}{c}\text { Terminal } \\
\text { outer } \\
\text { diameter } \\
(\mathrm{mm})\end{array}$} & \multicolumn{5}{|c|}{ Node } & \multirow{3}{*}{$\begin{array}{c}\text { Element } \\
\text { Stress } \\
(\mathrm{Mpa})\end{array}$} \\
\hline & \multicolumn{4}{|c|}{ Strain } & \multirow{2}{*}{$\begin{array}{l}\text { Stress } \\
(\mathrm{Mpa})\end{array}$} & \\
\hline & Direction X & Direction $Y$ & Direction Z & Total strain & & \\
\hline 18.25 & 0.002242 & 0.011072 & 0.014236 & 0.018537 & 58.6822 & 98.3998 \\
\hline 19.00 & 0.002101 & 0.011512 & 0.014801 & 0.019175 & 49.5758 & 97.5254 \\
\hline 19.75 & 0.002083 & 0.01337 & 0.014619 & 0.01909 & 49.6499 & 112.128 \\
\hline 20.50 & 0.001691 & 0.011512 & 0.011480 & 0.019175 & 61.639 & 113.563 \\
\hline 21.25 & 0.002077 & 0.011186 & 0.014381 & 0.018955 & 52.1436 & 83.0865 \\
\hline 22.00 & 0.002066 & 0.011213 & 0.014417 & 0.018895 & 52.894 & 68.1619 \\
\hline 22.75 & 0.002496 & 0.011318 & 0.014552 & 0.018858 & 67.647 & 70.4701 \\
\hline 23.50 & 0.002035 & 0.014502 & 0.014502 & 0.018837 & 67.1104 & 69.4118 \\
\hline 24.25 & 0.002496 & 0.011318 & 0.014552 & 0.018858 & 54.3026 & 70.4781 \\
\hline 25.00 & 0.002489 & 0.011357 & 0.014602 & 0.018893 & 68.4893 & 71.8922 \\
\hline 25.75 & 0.002479 & 0.011394 & 0.014649 & 0.018926 & 71.3153 & 73.5647 \\
\hline 26.50 & 0.002465 & 0.011427 & 0.014692 & 0.018958 & 89.8687 & 93.9635 \\
\hline 27.25 & 0.002635 & 0.011457 & 0.01473 & 0.019007 & 96.1249 & 114.998 \\
\hline 28.00 & 0.002631 & 0.011477 & 0.014756 & 0.019053 & 123.031 & 136.762 \\
\hline
\end{tabular}

Table.3.The calculation results of the model

According to the structure of anchor and applied load, axisymmetric element can be used to simulate and analyze.Compare the results of the element strain, element stress and joint stress to gain the data shown in the table below.Draw out the clip at the end of the outer radius and the anchor nodal stress, strain, stress with lines as shown below.

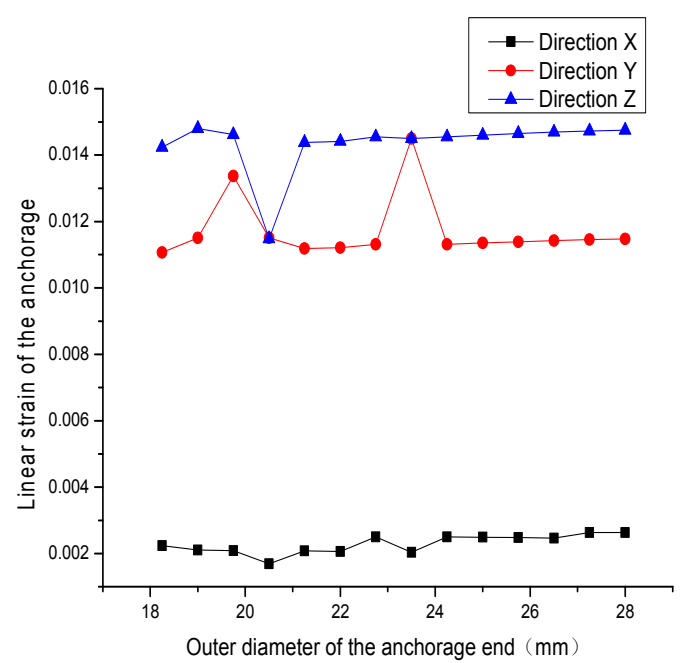

Fig.4.The linear strain of the anchorage

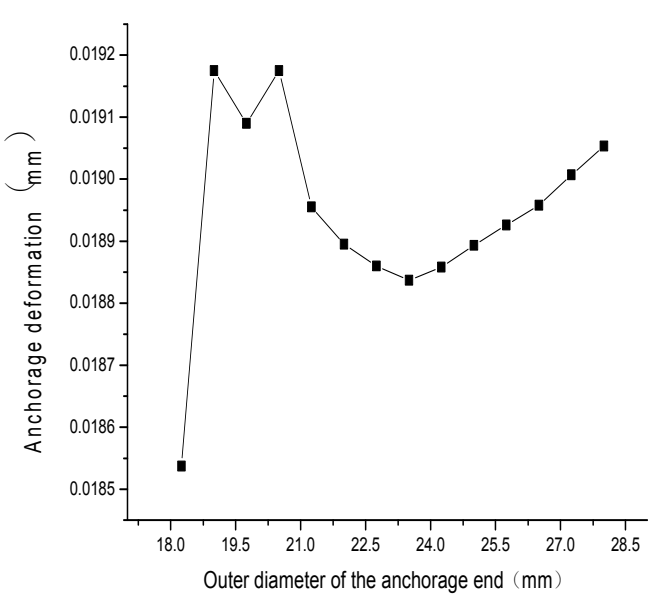

Fig.5.The anchorage deformation 


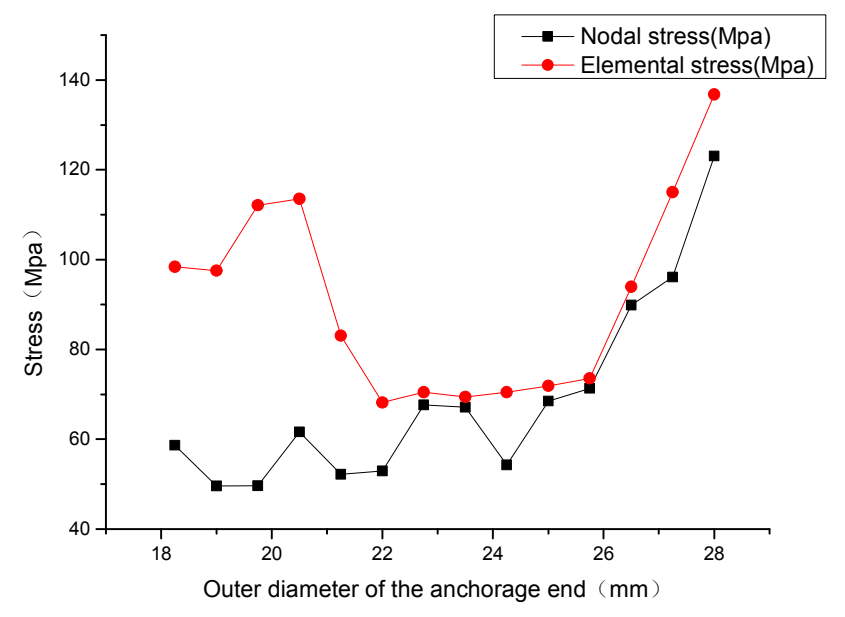

Fig.6.The stress

\section{Conclusion}

(a)Keep the constant parameters of the anchorage taper, plate length and plate thickness while change the anchor clamp sheet thickness then stress and strain of anchorage is transformed, which means the thickness of the anchor clip has impact on the bearing capacity of prestressed anchorage.

(b)With the increase of the thickness of the clip, the maximal anchorage elemental stress and nodal stress are increasing as a whole.Among them, the maximal nodal stress in the first half shows the steady growth.After $24.25 \mathrm{~mm}$, the stress rises remarkably. The maximal elemental stress shows upward tend in the first half interval but begins to decrease a little after $20.50 \mathrm{~mm}$.In $22.00 \mathrm{~mm}$ and its rear, the curves of maximal elemental stress and maximal nodal stress are rising in a same state.

(c)The strain which changes from different directions of anchorage is stable and the overall strain changes with the outer diameter of the anchor end to increase at first, then decrease slightly.For the diameter, after $23.50 \mathrm{~mm}$, anchorage by line of force of the strain and the end diameter is positively proportional relationship which also means that with the increasing diameter, linear strain will also increase.But when the outer diameter ranges from $21.25 \mathrm{~mm}$ to $25.75 \mathrm{~mm}$, the stress and the strain show a relatively stable trend.In the case of the strain control, there is a minimal strain when the outer diameter is $23.25 \mathrm{~mm}$ which is the excellent value of the simulation within a reasonable range.

(d)For the excellent value taken, it requires the anchor to fully meet the need of stretch-draw in the working field.According to the "national standard", the anchoring efficiency coefficient shall not be less than 0.95 and the total strain at fracture is not less than $2 \%$ [8]. The performance of fatigue stress meets the stress test of the national standard which is $65 \%$ of the strand's tensile strength for the upper limit and the number of cycles is 2 million times[7].

\section{Acknowledgement}

This project 11472005 supported by National Natural Science Foundation of China and Anhui Pr ovincial Science and Technology Research Project Funding through grant No.1501041133.

\section{References}

[1] Xie Qiang.Anchorage Stress Analysis and Structural Optimization of -ANSYS Finite Element simulation[D].Shanghai: Machinery Manufacturing and Automation, 2002 :231-244.

[2] Bao Chen,Wang Hujia.Examples of Advanced Engineering(Revised Edition) ANSYS Beijing: China Water Conservancy and Hydropower Press, 2009:340-360

[3] Zhou Minghua.Factors Influencing the Performance of the Anchorange with Wedged Jaws[J].Bridge Construction.2001 (2):45-47 
[4] Tao Hao, Duan Hongjie, Liu Jianxiu.Study on strength performance and destruction of prestressed anchor[J].Journal of Machine Design, 2003, 20 (8)13-15

[5] Guo Honglei,Jiang Yongsheng,Ding Dajun.The Crack Behavior of Anchorage Zone with the Internal Anchorage Tool[J]. Journal of Southeast University, 1996, 26 (6):70-74

[6] Kong Qinghua,Wang Siling,Xie Qiang.Structure analysis of wedge type single hole prestressed anchor[J].Shanghai:Grinder and Grinding2005,30:1-11

[7] Wang Guoyu, Liu Dongqing. Elastic-plastic finite element analysis of prestressed anchor[J].2005,24Journal of Liaoning Technical University(Suppl.)2005,24.96-98

[8] Chinese Research Institute of building science.GB/T14370-93. Anchorage, Grip and Coupler for Prestressing Tendons[M], Beijing:China Standard Press,1994.3-4 